\title{
Participación activa del alumnado en el proceso de enseñanza en Psicometría
}

\author{
Active participation of \\ students in the teaching \\ process in Psychometrics
}

SALVAdor CHACón Moscoso

ORCID: https://orcid.org/0000-0002-6401-7384

Universidad de Sevilla.

Facultad de Psicología

Departamento de Psicología

Experimental.schacon@us.es

Fecha de recepción: 19-11-2019

Fecha de aceptación: 25-11-2019

DOI: http://dx.doi.org/10.12795/9788447221912.080

Pp.: $1839-1850$ 
En las clases de Psicometría impartidas en el tercer curso del Grado en Psicología, el alumnado tiene una participación activa en las clases prácticas; sin embargo, en clases teóricas su actividad se reduce a escuchar la lección y preguntar posibles dudas que surjan. El objetivo de este Ciclo de Mejora en el Aula fue el de dotar al alumnado, también en clases teóricas, de un papel más activo con el fin de fomentar una adquisición de conocimientos más profunda. La tarea consistió en, con ayuda del profesorado, preparar parte de los contenidos de la materia y exponerlos en clase. Con esta metodología, la participación fue baja, y se encontró una adquisición de conocimiento media - alta, si bien los contenidos más puramente teóricos fueron los que se adquirieron en menor medida. Como mejoras para Ciclos futuros, se planea fomentar la participación y ampliar el peso de la teoría, valorando en mayor medida ambos aspectos en la calificación final de la asignatura.

Palabras Clave: Ciclo de Mejora en el Aula, Psicometría, aprendizaje activo, participación, teoría.

\section{Abstract}

In the Psychometrics classes taught in the third year of the Degree in Psychology, students have an active participation in the practical classes. However, in theoretical classes, their activity is reduced to listening to the lesson and asking possible doubts that arise. The objective of this Classroom Improvement Cycle was to provide students, also in theoretical classes, with a more active role in order to foster a deeper knowledge acquisition. The task consisted of, with the help of teachers, preparing part of the contents of the subject and presenting them in class. With this methodology, participation was low, and an acquisition of medium - high knowledge was found, although the most purely theoretical contents were those that were acquired to a lesser extent. As improvements for future Cycles, it is planned to encourage participation and increase the weight of the theory, valuing both aspects in a higher degree in the final grade of the subject.

Key words: "Classroom Improvement Cycle", "Psychometrics", "active learning", "participation", "theory" 


\section{Descripción del contexto}

La asignatura en que se llevó a cabo este Ciclo de Mejora en el Aula (CIMA) fue Psicometría, de tercer curso del Grado en Psicología, primer cuatrimestre; concretamente, fue en el grupo de inglés, donde todos los materiales estuvieron en inglés, al igual que las pruebas de evaluación, y tanto profesorado como alumnado hablaron en inglés. La principal ventaja de este grupo es que, por normativa, el número de matriculados no puede pasar de 40, mientras que en un grupo de español puede haber el doble de estudiantes.

Habitualmente, el alumnado asistía a clases teóricas presenciales donde el profesorado impartía los contenidos de los que luego se realizaban ejercicios en las clases prácticas. Esta asignatura no suele ser por la que el alumnado siente más motivación, dado que es del área de metodología, y lo más común es que los estudiantes aspiren a trabajar en otras áreas de contenido sustantivo (clínica, social, etc.).

En el curso anterior, mi intervención estuvo centrada en el uso en clase de ejercicios de formato cerrado (Chacón, 2018). Para complementar, este año me interesé en trabajar un nuevo método en las clases teóricas. Con los ejercicios de formato cerrado, se incrementó la motivación del alumnado para que trabajara en la asignatura. Precisamente lo mismo se esperaba, centrándonos en esta ocasión en la teoría.

Este curso cambié de asignatura (la intervención el curso pasado se ejecutó en Diseño y Análisis de Datos 1). Psicometría tiene puntos en común con ésta, dado que ambas son de la misma área y se trabaja comprensión de conceptos estadísticos y cálculos. Se esperaba por tanto que las conclusiones obtenidas el curso pasado sirvieran para el CIMA actual. Es de resaltar que el $80 \%$ del 
alumnado formó parte del estudio previo, ya que el alumnado que cursa las asignaturas en inglés se mantiene en los grupos de inglés hasta finalizar el grado.

\section{Modelo metodológico desarrollado}

En clases prácticas, el alumnado solía desempeñar un rol más positivo; sin embargo, en clases teóricas intervenían menos. Más bien tomaban apuntes y preguntaban las dudas que les iban surgiendo. Lo ideal sería que en clases teóricas el alumnado fuera tan activo como en las prácticas. Una forma de hacerles participar sería que prepararan parte del temario por su cuenta y que explicaran a otros compañeros lo que han aprendido (Porlán, 2017).

El objetivo de este CIMA fue potenciar la idea de una enseñanza basada en el manejo de la información, más que en la adquisición de conocimientos definitivos e inamovibles. Consideré que en el equipo docente éramos capaces de transmitir esta perspectiva de trabajo al dedicarnos al desarrollo de revisiones sistemáticas y meta-análisis.

Mi modelo metodológico ideal y que vi posible aplicar en este ciclo, planteó que el alumnado debía ser proactivo y colaborativo en el desarrollo de los contenidos de la materia. Disponer de prácticamente toda la información en internet, en bases de datos electrónicas y compartir espacios virtuales con el resto de alumnado posibilitó este modelo metodológico de trabajo (Chacón, 2015).

El pilar de mi modelo metodológico fue la colaboración del alumnado, dado que era quien desarrollaría los contenidos. Por lo tanto, este factor fue el que marcó hasta dónde pudo llegar la aplicación de esta intervención. A mayor motivación e implicación del alumnado, más se acercaría mi modelo posible al ideal. 
La Figura 1 muestra, de manera esquemática, el modelo seguido.

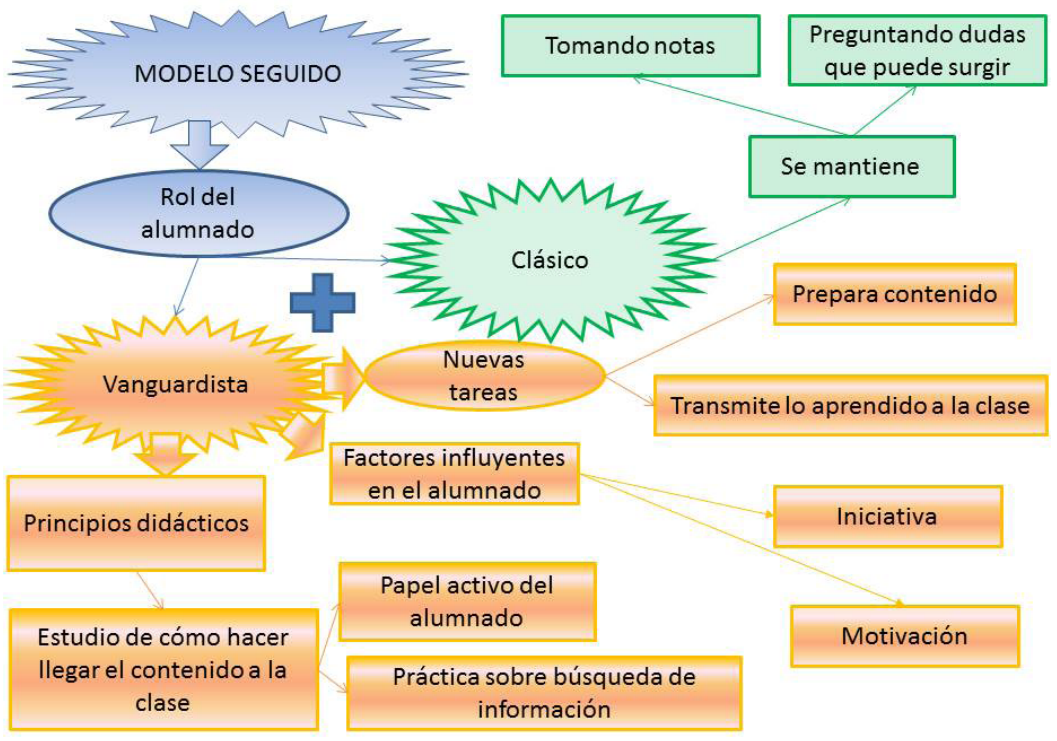

Figura 1. Representación de los modelos habitual, ideal y posible.

Jornadas de Formación e Innovación Docente del Profesorado | № 2 (2019) Esta obra se distribuye con la licencia Creative Commons Reconocimiento-NoComercial-SinObraDerivada 4.0 Internacional (CC BY-NC-ND 4.0.) 


\section{Mapa de contenidos y problemas}

La Figura 2 presenta la interconexión existente entre los diferentes temas tratados en la asignatura, de entre los cuales el alumnado pudo elegir uno para participar en las actividades de este ciclo de mejoras. Adicionalmente, se presentan las preguntas claves a responder, que sirvieron para centrar la atención en lo más relevante.

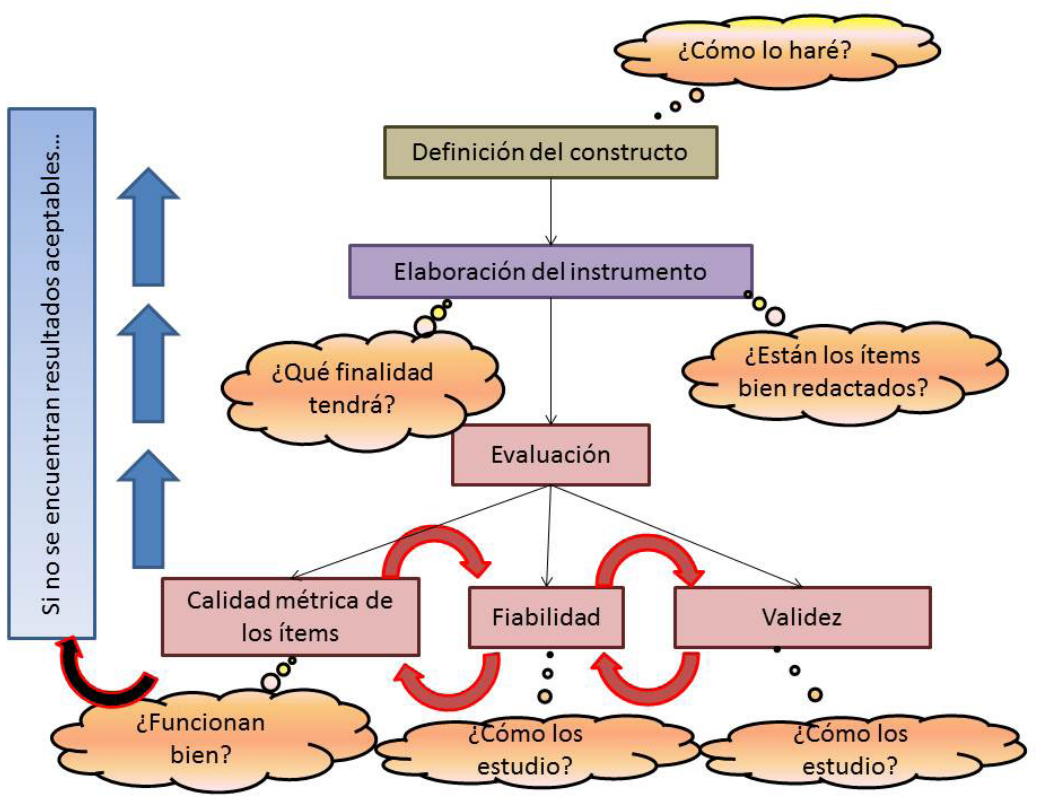

Figura 2. Interconexión entre los contenidos a tratar en la asignatura.

La secuencia de actividades fue la siguiente:

1. A principio de curso se le propuso al alumnado la posibilidad de prepararse una unidad didáctica del temario de la asignatura que supondría un incremento en su calificación final. Se indicó al alumnado que la exposición habría de estar encaminada en responder una de las preguntas claves incluidas en la Figura 2.

2. Vía correo electrónico o en una sesión presencial, el alumnado interesado planteó al profesor en su despacho los objetivos concretos de la 
actividad. Específicamente, se ofreció al alumnado interesado una guía de los pasos y calendarización de actividades que tuvieron que realizar hasta acabar llevando a cabo la presentación en clase, y una relación de preguntas tipo test para evaluar al resto del alumnado respecto a esa materia impartida.

3. El alumnado realizó un barrido bibliográfico para presentar a revisión por el profesor antes de iniciar la elaboración de la unidad didáctica.

4. El alumnado redactó la unidad didáctica con el apoyo del profesor.

5. El alumnado realizó una presentación de dicha unidad didáctica.

6. El alumnado realizó cinco preguntas tipo test de opción cerrada que respondió el alumnado de clase.

\section{Cuestionario inicial - final}

Partiendo de los contenidos a impartir en la asignatura, se aplicó un pre-test el primer día de clase que volvió a aplicarse ya avanzado el curso. Las preguntas fueron las siguientes:

1. ¿Qué finalidad puede tener un test psicológico?

2. ¿Qué estrategias seguirías para definir el constructo psicológico que pretendes medir?

3. ¿Existen constructos psicológicos con diferente grado de complejidad

4. Respecto al siguiente ítem:

"Estoy a favor de la cadena perpetua y de la pena de muerte $12345^{\prime \prime}$

¿Podrías mejorarlo? ¿Cómo?

5. ¿Qué características se estudian de un ítem para determinar su calidad psicométrica?

6. ¿Para qué se usa el alfa de Cronbach?

7. ¿Para qué se usa el análisis factorial? 
8. En un test psicológico, ¿son importantes las instrucciones de aplicación? ¿Por qué?

\section{Relato resumido de las sesiones}

Cada participante tuvo una fase de preparación y otra de exposición. Nadie pudo pasar a la fase de exposición sin haber pasado previamente por la de preparación. De este modo, se controló que los contenidos impartidos en clase por el alumnado fueran de calidad y carentes de errores. En las sesiones preparatorias, el profesorado dio algunas indicaciones generales sobre dónde encontrar información; posteriormente, el alumnado presentó un esquema de su exposición (en formato de presentación en PowerPoint) y las preguntas que posteriormente haría a sus compañeros. La sesión de exposición por parte del alumnado a su clase consistió en: (a) contextualización del contenido a impartir en el grueso de los contenidos de la asignatura; (b) explicitación de qué pregunta clave se iba a responder; (c) puesta en marcha de la exposición con apoyo de la presentación en PowerPoint realizada y revisada por el profesorado; (d) turno de preguntas por parte de la clase acerca de los contenidos expuestos; y (e) respuesta a las preguntas planteadas del alumnado a su clase, con el fin de obtener feedback del grado de conocimiento adquirido.

\section{Evaluación del aprendizaje de los estudiantes}

Con las respuestas que dio el alumnado, se elaboraron escaleras de aprendizaje, que sirvieron para detectar las mayores lagunas en el conocimiento previo del alumnado, con lo que se supo en qué incidir en mayor profundidad con las actividades a realizar. 
Se esperaron mejoras en las escaleras de aprendizaje, tras compararse las respuestas dadas en el pre-test con las obtenidas en el post-test. La Tabla 1 presenta los porcentajes de participantes que se ubicaron en cada uno de los niveles antes y después de la aplicación del CIMA. Además, se realizó un estudio inferencial con el estadístico X² de McNemar para determinar si las diferencias encontradas fueron estadísticamente significativas.

Tabla 1. Resultados de la comparación pre - post.

\begin{tabular}{|c|c|c|c|c|c|}
\hline № & Ítem & Respuestas $(N=25)$ & $\%$ pre & $\%$ post & $x^{2} / g l / p$ \\
\hline 1 & $\begin{array}{l}\text { Finalidad de } \\
\text { un test }\end{array}$ & $\begin{array}{l}\text { No responde } \\
\text { Diagnóstico } \\
\text { Selección y diagnóstico } \\
\text { Evaluación }\end{array}$ & $\begin{array}{l}4 \\
48 \\
44 \\
4\end{array}$ & $\begin{array}{l}12 \\
8 \\
40 \\
40\end{array}$ & $\begin{array}{l}20 \\
4 \\
<.001\end{array}$ \\
\hline 2 & $\begin{array}{l}\text { Estrategias } \\
\text { definición } \\
\text { constructo }\end{array}$ & $\begin{array}{l}\text { No responde } \\
\text { Incorrecto } \\
\text { Expertos y bibliométrico } \\
\text { Técnicas de Discusión }\end{array}$ & $\begin{array}{l}48 \\
40 \\
8 \\
4\end{array}$ & $\begin{array}{l}28 \\
48 \\
20 \\
4\end{array}$ & $\begin{array}{l}8 \\
2 \\
.018\end{array}$ \\
\hline 3 & $\begin{array}{l}\text { Constructos } \\
\text { con diferente } \\
\text { grado de } \\
\text { complejidad }\end{array}$ & $\begin{array}{l}\text { No responde } \\
\text { Respuesta incorrecta } \\
\text { Correcta parcialmente } \\
\text { Explicación extensa }\end{array}$ & $\begin{array}{l}20 \\
36 \\
40 \\
4\end{array}$ & $\begin{array}{l}16 \\
24 \\
32 \\
28\end{array}$ & $\begin{array}{l}9.667 \\
4 \\
.046\end{array}$ \\
\hline 4 & $\begin{array}{l}\text { Mejora ítem } \\
\text { con doble } \\
\text { pregunta }\end{array}$ & $\begin{array}{l}\text { No responde } \\
\text { Respuesta incorrecta } \\
\text { Añadir nuevos valores } \\
\text { Añadir nuevos valores y } \\
\text { dimensiones }\end{array}$ & $\begin{array}{l}20 \\
32 \\
44 \\
4\end{array}$ & $\begin{array}{l}8 \\
8 \\
36 \\
48\end{array}$ & $\begin{array}{l}21 \\
4 \\
<.001\end{array}$ \\
\hline 5 & $\begin{array}{l}\text { Característica } \\
\text { ítem (calidad } \\
\text { psicométrica) }\end{array}$ & $\begin{array}{l}\text { No responde } \\
\text { Respuesta incorrecta } \\
\text { Dificultad } \\
\text { Dificultad y discriminación } \\
\text { Dificultad, discriminación, } \\
\text { fiabilidad } \\
\text { Dificultad, discriminación, } \\
\text { fiabilidad, validez }\end{array}$ & $\begin{array}{l}44 \\
24 \\
16 \\
8 \\
4 \\
4\end{array}$ & $\begin{array}{l}4 \\
16 \\
12 \\
16 \\
28 \\
\\
24\end{array}$ & $\begin{array}{l}20 \\
11 \\
.045\end{array}$ \\
\hline 6 & $\begin{array}{l}\text { Uso alfa } \\
\text { Cronbach }\end{array}$ & $\begin{array}{l}\text { No responde } \\
\text { Respuesta incorrecta } \\
\text { Fiabilidad }\end{array}$ & $\begin{array}{l}84 \\
12 \\
4\end{array}$ & $\begin{array}{l}24 \\
20 \\
56\end{array}$ & $\begin{array}{l}17 \\
3 \\
.001\end{array}$ \\
\hline
\end{tabular}

Jornadas de Formación e Innovación Docente del Profesorado | № 2 (2019) Esta obra se distribuye con la licencia Creative Commons Reconocimiento-NoComercial-SinObraDerivada Internacional (CC BY-NC-ND 4.0.) 
A la luz de las notas tomadas en el procedimiento y de los resultados obtenidos en la comparación pre - post, se hace necesaria la instauración de nuevos hábitos en la práctica educativa para solventar algunas carencias detectadas. En primer lugar, para aumentar la participación, habría que dar un peso importante a la calificación de la actividad realizada sobre la calificación global. Se ve que, si es poca puntuación la que se obtiene, o esta puntuación se puede obtener a través de otra vía, el alumnado tiende a evitar la situación de tener que preparar un tema y presentarlo y defenderlo ante la clase

En segundo lugar, parece que el alumnado controla las cuestiones de procedimiento y cálculo, lo que les hace aprobar la asignatura y, sin embargo, adolece de conocimientos profundos sobre los contenidos teóricos y aplicaciones prácticas de lo aprendido. Por ello, se tomarán en el futuro medidas tales como: (a) realizar ensayos o resolución de casos donde el alumnado tenga que aplicar y redactar los conocimientos adquiridos en lugar de ceñirse a responder preguntas en formato cerrado, que es lo que actualmente se hace en la asignatura; y (b) preguntar en el examen más cuestiones teóricas.

Jornadas de Formación e Innovación Docente del Profesorado | № 2 (2019) Esta obra se distribuye con la licencia Creative Commons 


\section{Referencias bibliográficas}

Chacón, S. (2015, julio). Programas informáticos: un pilar clave en la enseñanza de la metodología en la actualidad. II Jornadas de Docencia Universitaria. Sevilla: Facultad de Ciencias de la Educación de la Universidad de Sevilla. ISBN: 978-84-88359056.

Chacón, S. (2018). Trabajo en grupo en base a ejercicios de formato cerrado. En V Jornadas de Docencia Universitaria (p.p. 146-160). Sevilla: Instituto de Ciencias de la Educación de la Universidad de Sevilla. Disponible en http://dx.doi.org/10.12795/JDU.2018.i01.08

Porlán, R. (2017). Enseñanza universitaria. Cómo mejorarla. Madrid: Morata. 